\title{
Simultaneous method for the separation and identification of certain benzodiazepine drugs in pharmaceutical formulations by liquid chromatography-tandem mass spectrometry (LC- $\mathrm{MS} / \mathrm{MS})$
}

\begin{abstract}
Background: The Benzodiazepine (BZD) drugs are frequently encountered in clinical and forensic casework samples involving road traffic offences and/or drug overdoses. An overdose of BZD can cause respiratory depression, coma and death. A sensitive and selective method for the simultaneous method for the separation and identification of certain abused BZDs viz. diazepam, lorazepam, nitrazepam, chlordiazepoxide, flurazepam, alprazolam, etizolam, clobazam, clonazepam, and midazolam drugs was developed together with a qualitative screening in pharmaceutical formulations.
\end{abstract}

Methodology: The instrument was operated using an electrospray ionization mode (ESI) in combination with the C18 reverse phase column (120 A, 2.1 x $150 \mathrm{~mm}$ Acclaim 120) 4.5 minutes resulted in the best separation and was selected. Chromatographic separation was performed with a different solvent combinations were tested to achieve the best separation. The mobile phase consisted of a mixture of methanol and $20 \mathrm{mM}$ ammonium formate (50: $50, \mathrm{v} / \mathrm{v}$ ) adjusted $\mathrm{pH} 8.6$. A run of $25 \mathrm{~min}$, including a re-equilibration time of $10 \mathrm{~min}$ was selected.

Results: The most implicated drug class was BZDs, either alone or in combination with alcohol. In this report, a procedure was developed for the screening of ten BZDs in pharmaceutical formulations by LC-MS/MS. The limit of detection ranged from $25-250$ $\mu \mathrm{g} / \mathrm{mL}$ for all BZDs. Our results demonstrate the suitability of the LC-MS/MS method for the simultaneous separation and identification of BZDs, and new psychoactive substances in forensic samples.

Recommendations: The results of the study showed that the proposed LC-MS/MS method is simple, rapid, precise and accurate, which is useful for the separation and identification of BZD drugs in forensic, clinical analytic toxicology and might be useful for the analyses of suspected drug-facilitated sexual assault (DFSA) cases.

Keywords: forensic science, benzodiazepine, separation, detection, pharmaceutical formulations
Volume 5 Issue I - 2017

\section{Subbiah Thangadurai, Bose Karthikprabu, Arulraj Tamilarasan \\ Post Graduate Studies and Research, Department of Chemistry Raja Doraisingam Government Arts College, India}

Correspondence: Subbiah Thangadurai, Post Graduate Studies and Research, Department of Chemistry Raja Doraisingam Government Arts College,Sivagangai - 630 56I, Govt. of Tamil Nadu, India, Tel 91-9488054919, Email www.rdgacollege.in.

Received: April 21, 2017| Published: June 08, 2017
Abbreviations: LC-MS/MS, liquid chromatography-tandem mass spectrometry; BZD, benzodiazepine; GC, gas chromatography; ESI, electro spray ionization; DFSA, drug-facilitated sexual assault, DFC, drug-facilitated crime

\section{Introduction}

Forensic science has been developed in the twentieth century and came to be recognized by the courts and law enforcement. Forensic analysis and investigation capabilities are vital in criminal and civil cases, national security, environmental protection, and public safety. The manufacture and trafficking of illicit drugs and counterfeiting of legitimate pharmaceuticals, new emerging challenges from organized crime and terrorist groups, also underline the vital need for sensitive and selective rapid identification of substances such as explosives and toxic spills in field, drug mixture. Presently, practical analytical technologies are needed to enable the "in field" screening and analysis of evidence to provide fast, accurate, scientific information to support the forensic investigation. ${ }^{1}$ Forensic analytical chemistry is defined as a discipline applied to crime scene analysis and to law and is one of the areas of analytical chemistry where the nature of the sample and the use of the analytical chemical information play important roles in selecting and executing the appropriate chemical analysis technique. This part of forensic sciences deals with the characterization and quantitation of chemical substances at trace levels. Most forensic samples are complex mixtures for which analysis generally requires separation prior to identification of chemical species. Thus, the principal tools of the forensic chemist are the instruments of analytical chemistry, with emphasis mainly on chromatographic techniques i.e., Thin Layer Chromatography (TLC), Gas Chromatography (GC) and Liquid Chromatography (LC) with the help of many other hyphenated 
techniques. The Benzodiazepines (BZDs) are widely known as anxiolytics, sedative hypnotics, anticonvulsants, or muscle relaxants. ${ }^{2}$ Clinical characteristics of BZDs include a wide safety margin of their therapeutic index and minimal serious adverse side effects. The advent of numerous new psychoactive substances is a challenge for clinical toxicology and drug testing laboratories. ${ }^{3}$ The increasing number of these samples has created a need for higher sample throughput, requiring faster sample preparation and analysis. BZDs are widely used to aid patients with anxiety and sleeping disorders and, hence, have potential for abuse. For most countries, the BZDs are classified as controlled drugs, and yet they are frequently encountered in clinical and forensic toxicological analyses involving intoxication, over dosage, and traffic accidents and are sometimes implicated in the commitment of crimes. The determination of various abused drugs is important in many fields of analytical toxicology, such as forensic analysis and workspace drug testing. A forensic toxicology analytical method must provide rapidity, simplicity, high reliability, and accuracy for screening drugs and metabolites of toxicological interest in suspected biological samples. In addition, abuse of BZDs and some related substances was found to be associated with suicide or drug-facilitated sexual assault (DFSA). In many cases, the DFSA victims might not report the event and receive medical attention until several hours or days after the incident.

Drug-facilitated crime $(\mathrm{DFC})^{4}$ is a general term that includes rape or other sexual assault, robbery, money extortion, as well as the deliberate maltreatment of the elderly or children under the influence of psychotropic substances. DFC are criminal acts carried out by means of administering a substance to a person with the intention of impairing behaviour, perceptions or decision-making capacity. It also extends to taking advantage of an impaired person, without their consent, after their voluntary intake of an incapacitating substance. While the covert use of drugs to facilitate crime has occurred over the centuries, it has been recently been highlighted by a significant increase in reports of DFC worldwide. The DFSA, ${ }^{5}$ which is a subset of DFC, occurs when a person (male or female) is subjected to sexual act(s) while they are incapacitated or unconscious due to the effect(s) of ethanol, a drug and/or other intoxicating substance, and as a result unable to resist or consent to such acts. Substances may be administered covertly to an intended victim or victims, or a perpetrator may take advantage of a victim after voluntary ingestion of the substance. The use of the term "date rape" by the media in cases of sexual assault, to describe DFSA, may be misleading. The media has focused on only a few of the drugs such as Rohypnol ${ }^{\circledR}, \mathrm{GHB}$ and ketamine that can be used in DFSA. ${ }^{5}$ However, there are many other substances that can be used to facilitate such crimes including alcohol, over-the-counter medicines, other psychoactive prescription drugs and illicit substances. The abuse or misuse of BZDs is internationally widespread which means that any forensic laboratory may encounter a range of these compounds. In general, BZDs encountered in the illicit market are diverted from legitimated sources. The BZDs are currently by the far most important and most widely used anxiolytic drugs and some compounds are as sedato-hypnotics, anticonvulsants, muscle relaxants or anesthetics. The BZDs can also amplify the depressant effects of other drugs, including alcohol and opiates, so increasing the risk of overdose in polysubstance use. In this study, we have chosen ten of the BZDs viz. Diazepam, lorazepam, nitrozepam, chlordiazepoxide, flurazepam, alprazolam, etizolam, clobazam, clonazepam, and midazolam drugs, each having different structural characteristics (Figure1).

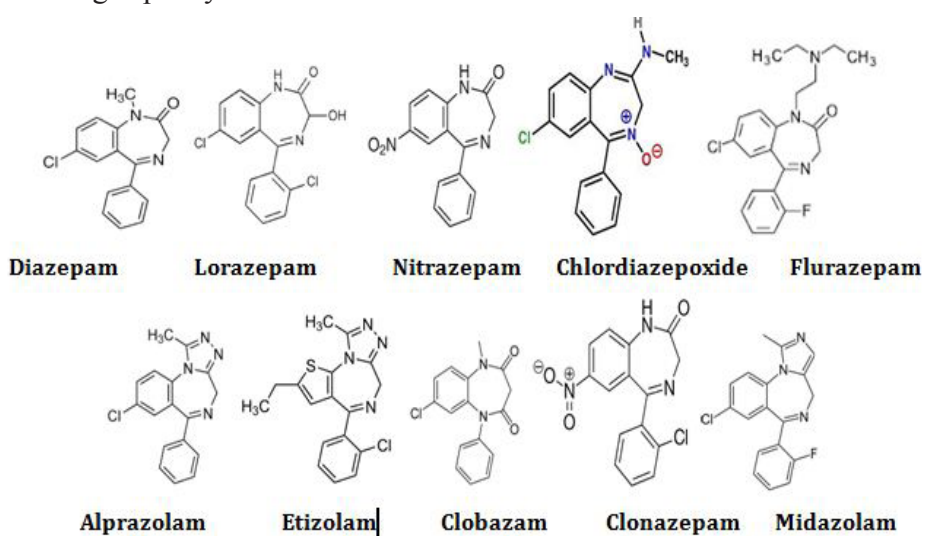

Figure I The structure of the studied ten Benzodiazepines.

These compounds are the most commonly prescribed class of drugs in the world for the treatment of anxiety and insomnia, particularly for the elders. ${ }^{6}$ Alprazolam is also a popular drug of abuse, it is of particular interest, because it is being prescribed for treatment of depression and has been implicated in suicidal ingestions. ${ }^{7}$ Alprazolam, and nitrazepam are used with low doses, and their therapeutic plasma concentrations are rather low. Chlordiazepoxide is used to treat anxiety and acute alcohol withdrawal. It is also used to relieve fear and anxiety before surgery. Chlordiazepoxide is utilised widely as tranquillisers, hypnotics, sedatives, antidepressants, for both humans and animals, which act by increasing the efficiency of the neurotransmitter $\gamma$-aminobutyric acid (GABA) to decrease the communication between neurons, so calming many of the functions of the brain. ${ }^{8}$ Diazepam, first approved for use in the early 1960s, is one of the most frequently prescribed drugs of the BZD group. Its uses include; treatment of anxiety and anxiety related insomnia, muscle relaxant, anti-epileptic and preoperative sedative. Therefore, the availability of reliable, sensitive, specific, and fast analytical methods for their determination is deemed important.

Clobazam has been used as an anxiolytic and in the treatment of epilepsy, and it is considered as a relatively safe drug. Paula Proencaa et al., ${ }^{9}$ has presented a fatal case with a 49 -year-old female, found dead at home. She had been undergoing psychiatric treatment and was a chronic alcoholic. The autopsy findings were unremarkable, except for multi visceral congestion, steatosis and a small piece of a plastic blister pack in the stomach. The clobazam was responsible for 
the death, ${ }^{10}$ probably by respiratory depression. Several methods ${ }^{11,12}$ for the determination of clobazam have been published. Gowri Bala Kumari et al., ${ }^{13}$ have developed and validated a simple, precise and accurate Reversed Phase High-Performance Liquid Chromatography (RP-HPLC) method for rapid assay of clobazam in tablet dosage form. Gazdzik et al. ${ }^{14}$ has described a method for simultaneous determination of Clobazam and its active metabolite $\mathrm{N}$-desmethylclobazam in various biological samples by RP-HPLC method with UV detection. The determination of both clobazam and N-desmethylclobazam was performed without derivatization. Clonazepam, a nitrobenzodiazepine and a scheduled drug, is one of the highly abused drugs in recent times. Idris et al., ${ }^{15}$ has developed an analytical methodology for the detection and quantitation of clonazepam in chocolate sample. Several methods ${ }^{16-18}$ have been described for analysing clonazepam.

AboulEnein et al., ${ }^{19}$ have described an accurate and reproducible method for the analysis of flurazepam hydrochloride in pharmaceutical preparations. It was a simple and rapid isocratic HPLC elution method was employed, which requires about 15 minutes to be performed. Dammalapati et al., ${ }^{20}$ have reported a new, sensitive, fast, precise, RPHPLC method for the determination of flurazepam in (capsule) dosage form. Dadgar et al., ${ }^{21}$ have developed a method for the determination of flurazepam and its metabolites in human blood plasma. Selinger et al. ${ }^{22}$ described a sensitive isocratic HPLC method, which allows the precise and accurate quantification of flurazepam and four metabolites with a single determination. Mehta ${ }^{23}$ had reviewed the methods available to date for the determination of chlordiazepoxide, clonazepam, diazepam, flurazepam, lorazepam, nitrazepam, oxazepam and their metabolites in biological fluids. The pharmacokinetics and bioavailability of these compounds and their concentrations in serum in cases of abuse, forensic cases, drug poisoning or suicidal excessive doses have been reported. ${ }^{24}$ Dordevic et al. ${ }^{25}$ have reported the use of photodiode array (HPLC-PDA) and mass spectrometric (LCMS) detection for determination and confirmation of midazolam in biological samples in therapeutic or toxic concentration. Nishiyama et al., ${ }^{26}$ have reported two cases of overdoses of intramuscular midazolam used as a premedication. Both cases had no resedation or complications, but the accidents happened because of a resident and nurse's lack of experience with midazolam. The postmortem tissue midazolam concentration reported in a death caused by self-injection of midazolam and sulfentanil. Michalodimitrakis et al., ${ }^{27}$ have reported midazolam related death of a 63 -year-old man that occurred during endoscopic retrograde cholangiopancreatography, after receiving 10 mg of midazolam.

The acute intoxication due to midazolam overdose was confirmed by HPLC analysis. For decades, HPLC has been the cornerstone of comprehensive drugs screening in forensic toxicology. Famiglini et al. ${ }^{28}$ have determined and quantified of BZDs in very small residues of beverages, collected at the scene of DFC are mandatory in legal procedures. Xiang et al. ${ }^{29}$ have frequently observed that the most BZDs are involved in the cases of DFC, a sensitive, specific, and reproducible methods for the quantitative determination of 18 BZDs in hair have been developed using LC-MS/MS. Morini et al., ${ }^{30}$ have developed and validated a multi-analyte method for the detection and quantification of 87 psychoactive drugs (antidepressants, antipsychotics, benzodiazepines, and z-drugs) in human hair using the LC-MS/MS. Due to the remarkable increase in requests of hair sample tests (such as for driver's license renewals, child custody, DFA cases, and postmortem toxicology), they have focused on the development of a rapid sample preparation. However, the LC-MS/MS method has been successfully applied for the analysis of postmortem cases.

In literature, several methods for the analysis of BZDs are described, generally consisting of GC-MS methods as well as methods using HPLC-DAD. However, HPLC with mass spectrometric detection is becoming increasingly the method of choice for simultaneous analysis and confirmation of many polar and/or chemically instable drugs, including BZDs. ${ }^{31,32}$ The BZDs and zolpidem are generally prescribed as sedative, hypnotics, anxiolytics or anticonvulsant drugs, however, are frequently misused in DFC. Paeng et al., ${ }^{33}$ have developed a rapid and simple liquid chromatography-tandem mass spectrometric (LC-MS/MS) method for identification and quantification of BZDs, zolpidem and their metabolites in urine using deuterium labeled internal standards (IS). Xiong et al., ${ }^{34}$ have developed a semi-automated method for the determination of four opioids and two-solid-phase extraction (CS-SPE) and LC-MS/MS. A simple and reliable analytical method was established and validated by Jang et al., ${ }^{35}$ for the simultaneous determination of twenty-five BZDs and zolpidem in oral fluid obtained using the Quantisal ${ }^{\mathrm{TM}}$ collection device. Nowadays, LC-MS is the method of choice for the analysis of drugs of abuse. A simultaneous screening method for multiple types of drugs has also become popular recently. However, because qualitative determination remains important in forensic science, ${ }^{32} \mathrm{GC}$ MS is still in use even if it requires complicated specimen preparation and derivatization procedures. This is because GC-MS is reliable and has been employed ever since for the appraisal of trials.

LC-MS has become very popular and today, it is the leading tool for the simultaneous multi component analysis of drugs of abuse. LC-MS is ideally suited for BZD family of compounds, because the technique does not require derivatization thereby saving time, expense and experimental difficulty. In this study, different types of mobile phases were studied on diazepam, lorazepam, nitrozepam, chlordiazepoxide, flurazepam, alprazolam, etizolam, clobazam, clonazepam, and midazolam drugs. There was lacking in published LC methods that are applicable to simultaneous separation and detection of BZD. For these reasons, the analysis of BZDs is of great interest to forensic and clinical toxicologists. The identification and confirmation of drugs of abuse requires an analytical technique that is highly sensitive and subjective of the different drugs. Gas chromatography mass spectrometry (GC-MS) was used for determination of various abused drugs in biological matrixes. ${ }^{36,37}$ The GC-MS technique was the most commonly used technique to separate and determine abused drugs and their metabolites due to its cost, high specificity, and sensitivity.

However, GC-MS analysis required a derivatization process to increase the volatility of many polar analytes and the derivatization step is costly, time-consuming, and susceptible to errors. There is a preference for using LC coupled with MS. Reports of screening or detecting abused drugs, BZDs, and new psychoactive substances using LC tandem mass spectrometry (LC-MS/MS) in multiple reaction monitoring (MRM) mode are available, ${ }^{3,38}$ however, there are still no reported studies concerning simultaneous identification of multiple abused drugs, benzodiazepines, and new psychoactive substance in pharmaceutical formulations by LC-MS/MS. The aim of this study was to develop a universal, rapid, and sensitive Liquid Chromatographic-Mass Spectrometric (LC-MS/MS) method for the simultaneous separation and identification of diazepam, lorazepam, nitrozepam, chlordiazepoxide, flurazepam, alprazolam, etizolam, clobazam, clonazepam, and midazolam drugs in bulk powder and 
in pharmaceutical dosage form, forensic laboratories as well as in confiscated materials. However, since the introduction of robust interfaces there has been a tremendous increase in the popularity of the technique amongst scientists from a wide variety of disciplines. LC-MS/MS has evolved into a robust and reliable tool that also offers versatility, specificity and sensitivity.

\section{Materials and methods}

\section{Samples}

Benzodiazepine drugs viz. diazepam, lorazepam, nitrazepam, chlordiazepoxide, flurazepam, alprazolam, etizolam, clobazam, clonazepam, and midazolam drugs were of pharmaceutical grade (India). The solvent methanol used was of HPLC Grade. The solvents were purchased from Qualikem Fine Chem Pvt. Ltd., New Delhi, India. Freshly prepared solutions were always employed.

\section{Preparation of solutions}

Preparation of standard solutions: Standard solutions of diazepam, lorazepam, nitrazepam, chlordiazepoxide, flurazepam, alprazolam, etizolam, clobazam, clonazepam, and midazolam drugs were prepared by taking $5 \mathrm{~mL}$ stock solution of each drug with a calibrated pipette and placing them in a $25 \mathrm{~mL}$ volumetric flask. The flask was filled with methanol to get the desired concentrations.

Preparation of stock solutions: Stock solutions of $1 \mathrm{mg} / \mathrm{mL}$ of diazepam, lorazepam, nitrazepam, chlordiazepoxide, flurazepam, alprazolam, etizolam, clobazam, clonazepam, and midazolam drugs were prepared. It was dissolved in an appropriate amount of the methanol solution, and stirred using a magnetic stirrer for a period between 15-30 min until it was completely dissolved.

Preparation of working solutions: Working solutions of 250, 150,100 and $50 \mu \mathrm{g} / \mathrm{mL}$ diazepam, lorazepam, nitrazepam, chlordiazepoxide, flurazepam, alprazolam, etizolam, clobazam, clonazepam, and midazolam drugs were prepared separately. These were freshly prepared for every experiment.

Preparation of sample solution: Diazepam, lorazepam, nitrozepam, chlordiazepoxide, flurazepam, alprazolam, etizolam, clobazam and clonazepam drugs both tablet and capsule samples were prepared separately by weighing 20 tablets individually and the average weight per tablet was calculated. The tablets were ground to get a fine powder. The powder equivalent to $30 \mathrm{mg}$ of clonazepam, clobazam, and flurazepam, were weighed and placed in a $100 \mathrm{~mL}$ volumetric flask. The powder was dissolved with methanol and mixed thoroughly on a magnetic stirrer for half an hour. The flask was filled with methanol and mixed. The solution was then filtered through Buchner's funnel with $0.45 \mathrm{~mm}$ filters. $50 \mathrm{~mL}$ of filtrate was placed into a $100 \mathrm{~mL}$ volumetric flask and was filled with methanol to obtain $1 \mathrm{mg} / \mathrm{mL}$ solution of clonazepam, clobazam and flurazepam. Midazolam ampoule samples were prepared from $(50 \mathrm{mg} / 10 \mathrm{~mL})$ ampoule solution. The ampoule solution was mixed with methanol for $5 \mathrm{~min}$ and $3 \mathrm{~mL}$ of solution was placed into a $100 \mathrm{~mL}$ volumetric flask. The volume was filled with methanol and mixed well to obtain $1 \mathrm{mg} / \mathrm{mL}$ solution of midazolam.

\section{LC-MS/MS analysis}

An LC-MS/MS system was with micrOTOF-Q II, Bruker, Germany. The sample was analyzed by LC/ESI/MS/MS using UHPLC+ focused (Ultra high performance liquid chromatography) RP liquid chromatography coupled to mass spectrometer. The data acquisition and processing software was designed by micrOTOF-Q II, Germany on Pentium computer. Instrumental operation conditions are given in (Table 1). LC-MS/MS was operated in positive ionization mode. Flow injection analysis was carried out with the standards of certain BZDs to optimize the mass parameters; Declustering potential (DP), Entrance potential (EP), Collision cell EP (CEP), Collision energy (CE), and collision cell exit potential (CXP).

Table I LC-MS/MS operational parameters

\begin{tabular}{llll}
\hline LC Conditions & \multicolumn{3}{l}{ MS Conditions } \\
\hline Column & $\mathrm{Cl} 8$ reverse phase column $(120 \AA$ A $2.1 \times$ I50 mm Acclaim I20) & lonization mode & ESI Positive \\
Column temp & $35^{\circ} \mathrm{C}$ & Drying gas flow & $4.01 / \mathrm{min}$ \\
Mobile Phase & Methano: $20 \mathrm{mM}$ ammonium formate $\mathrm{pH}=8.6(50: 50: \mathrm{v} / \mathrm{v})$ & Drying gas temp & $180^{\circ} \mathrm{C}$ \\
Flow rate & $0.5 \mathrm{~mL} / \mathrm{min}$ (optimized for the separation) & Nebulizer & $10.2 \mathrm{psi}$ \\
Injection volume & $10 \mu \mathrm{L}$ & Mass range & $\mathrm{m} / \mathrm{z} 50-3000$ \\
Post time & $4.5 \mathrm{~min}$ & Vcap & $4500 \mathrm{~V}$ \\
Run time & $25 \mathrm{~min}$ & Collision Cel RF & $150.0 \mathrm{VPP}$ \\
\hline
\end{tabular}

\section{Result and discussion}

Liquid chromatography separations were carried out on a C18 reverse phase column (UHPLC + Ultimate 3000 series, Dionex). With different solvent combinations were tested to achieve the best separation. The methanol/formic acid gradient in combination with the $\mathrm{C} 18$ reverse phase column (120 $\AA, 2.1$ x $150 \mathrm{~mm}$ Acclaim 120) 4.5 minutes PS resulted in the best separation and was selected. Figures 2(i) \& (ii) shows the overlaid ion chromatograms of the tested BZDs viz. diazepam, lorazepam, nitrozepam, chlordiazepoxide, flurazepam, alprazolam, etizolam, clobazam, clonazepam and midazolam drugs. A run of $25 \mathrm{~min}$, including a re-equilibration time of $10 \mathrm{~min}$ was selected. In GC-MS, as the ionization energy is rather constant, the $70 \mathrm{eV}$ EI mass spectra are rather uniform and unique for a molecule. On the other hand, in LC-MS the fragmentation largely depends on the geometric configuration of the LC-MS interface and on the chromatographic conditions applied, so that the spectra obtained differ widely with the nature of the eluent and the MS conditions. 

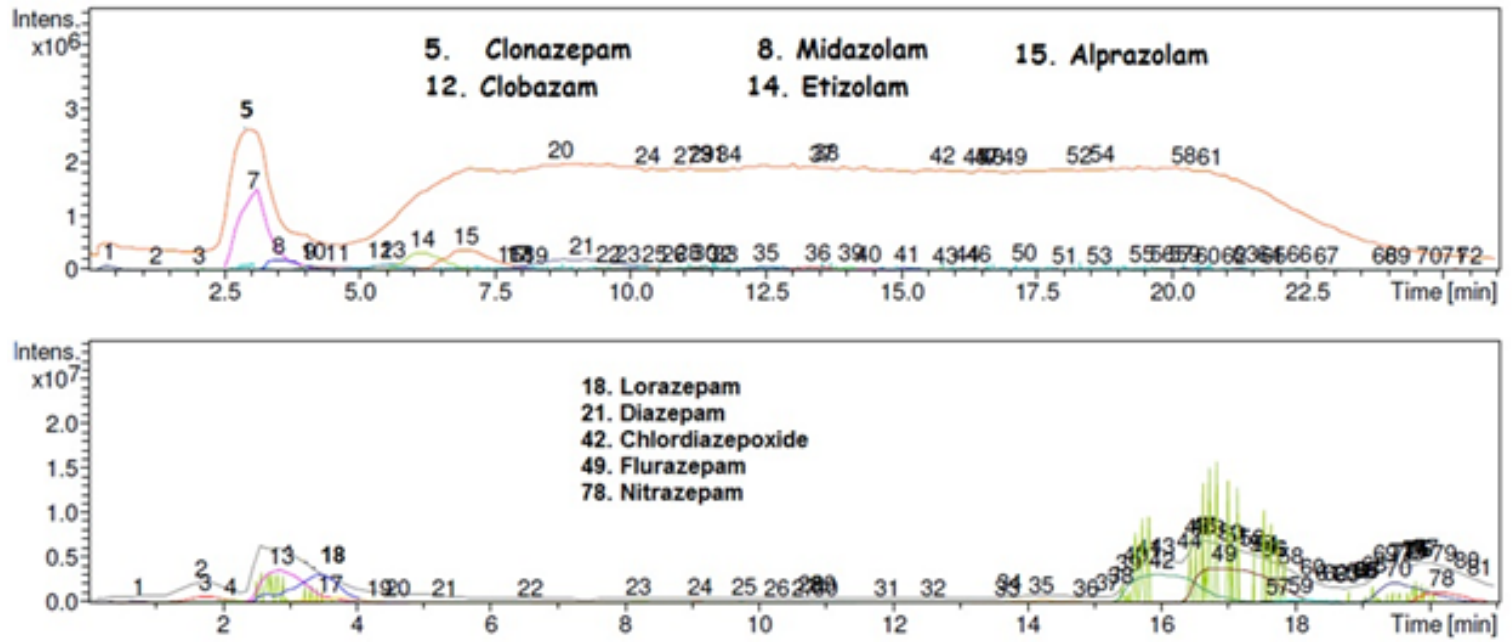

Figures 2(i)\&(ii) Overlay of principal product ion chromatograms of BZDs.

No single optimum ionization voltage can be set for a wide variety of compounds of forensic and doping interests as their fragmentation curves in ESI is showing quite different patterns. ${ }^{39}$ Even when the ionization energies are the same, LC-MS mass spectra appear in practice to be laboratory and instrument specific. Due to restrictions of the equipment in MS-MS mode concerning the number of scans per segments, it was not possible to use the MS-MS mode for all tested substances. The MS-MS product ion scan mode was used for the BZDs with low concentrations and for BZDs that co-elute. The alternate detection mode reduces the sensitivity of the detection. Therefore, the sensitivity was optimized by dividing the chromatographic run into segments. The segments were chosen in such a way that each contained a small number of BZDs at low concentrations. The choice of the segments allows for small fluctuations in the retention times.
The final acquisition mode used MS-MS detection for ten BZD drugs, were identified by the presence of the characteristic ions and listed in Tables 1 \& 2. The Figure 2 (i) \& (ii) shows the overlaid ion chromatograms of the tested BZD drugs. The (Figures 3-12) were shown MS-MS detection for BZD drugs. The ESI was evaluated for the analysis of these compounds. Generally, both gave good sensitivity at the low therapeutic levels needed for the method. However, both Clobazam and Clonazepam showed poor response in ESI. With the mobile phase which gave the best chromatographic separation in a reasonable time. Optimisation of chromatographic conditions are intended to take into account the various goals of the method development and to weigh each goal (resolutions, run time, sensitivity, peak symmetry, etc) accurately, according to the requirements of HPLC can be used for the estimation of BZDS in pharmaceutical and forensic samples.

Table 2 Mass spectral parameters (LC-MS/MS)

\begin{tabular}{|c|c|c|c|c|c|c|c|c|c|}
\hline S. No & BZD Drugs & $\begin{array}{l}\text { Retention } \\
\text { Time (Min) }\end{array}$ & $\begin{array}{l}\text { MS Molecular } \\
\text { Ion }\end{array}$ & $\begin{array}{l}\text { Acquisition } \\
\text { Mode }\end{array}$ & $\begin{array}{l}\text { MS/MS Precursor/ } \\
\text { Parent Ion }\end{array}$ & \multicolumn{3}{|c|}{$\begin{array}{l}\text { MS or MS-MS } \\
\text { Product Ions }\end{array}$} & \multirow{2}{*}{$\begin{array}{l}\begin{array}{l}\text { Scan } \\
\text { Range } \mathbf{~} / \mathbf{z}\end{array} \\
50-3000\end{array}$} \\
\hline I & Alprazolam & 7 & 309 & MS-MS & 308 & 223 & 209 & 182 & \\
\hline 2 & Clonazepam & 2.9 & 316 & MS-MS & 316 & 289 & 253 & 225 & $50-3000$ \\
\hline 3 & Clobazam & 5.4 & 301 & MS-MS & 301 & 284 & 249 & 239 & $50-3000$ \\
\hline 4 & Etizolam & 6.2 & 343 & MS-MS & 343 & 231 & 217 & 183 & $50-3000$ \\
\hline 5 & Midazolam & 3.5 & 326 & MS-MS & 326 & 261 & 195 & 166 & $50-3000$ \\
\hline 6 & Chlordiazepoxide & 16 & 300 & MS-MS & 300 & 285 & 284 & 283 & $50-3000$ \\
\hline 8 & Flurazepam & 16.9 & 388 & MS-MS & 388 & 360 & 302 & 194 & $50-3000$ \\
\hline 9 & Lorazepam & 3.6 & 321 & MS-MS & 321 & 163 & 142 & - & $50-3000$ \\
\hline 10 & Nitrazepam & 20.1 & 282 & MS-MS & 282 & - & - & & \\
\hline
\end{tabular}

Citation: Thangadurai S, Karthikprabu B, Tamilarasan A. Simultaneous method for the separation and identification of certain benzodiazepine drugs in pharmaceutical formulations by liquid chromatography-tandem mass spectrometry (LC-MS/MS). Forensic Res Criminol Int J. 20I 7;5(I):202-2I0. DOI: 10.15406/frcij.2017.05.00140 


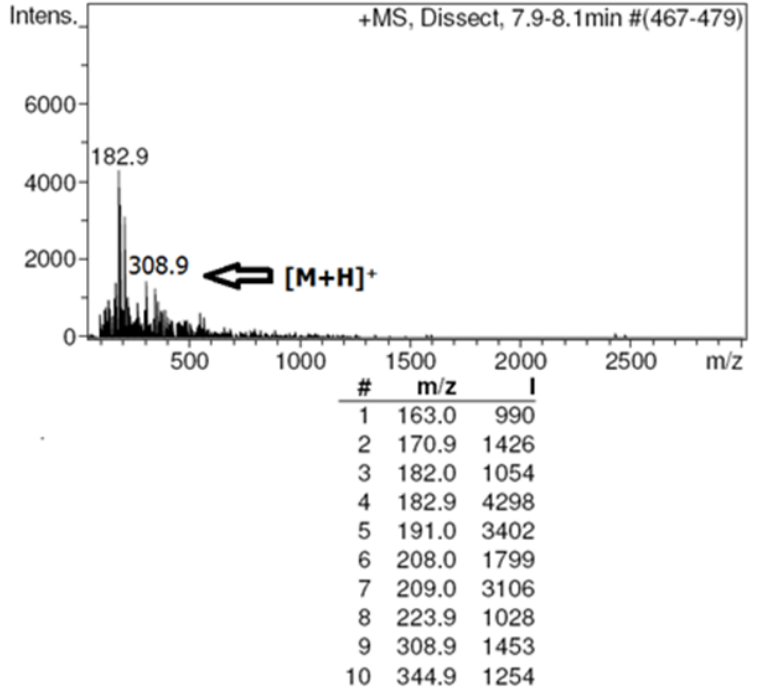

Figure 3 Mass spectra of alprazolam at retention time $7.0 \mathrm{~min}$.

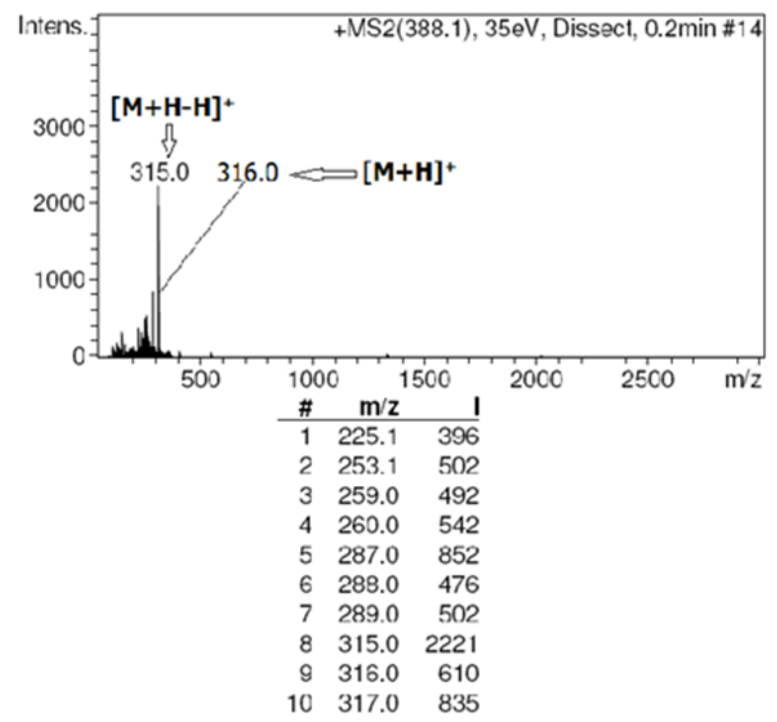

Figure 4 Mass spectra of clonazepam at retention time $2.9 \mathrm{~min}$.

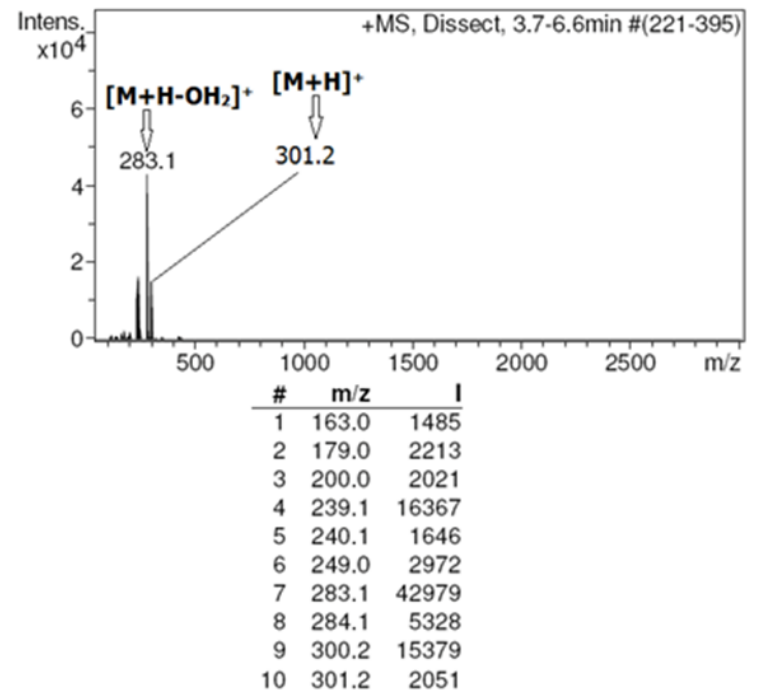

Figure 5 Mass spectra of clobazam at retention time $5.4 \mathrm{~min}$.

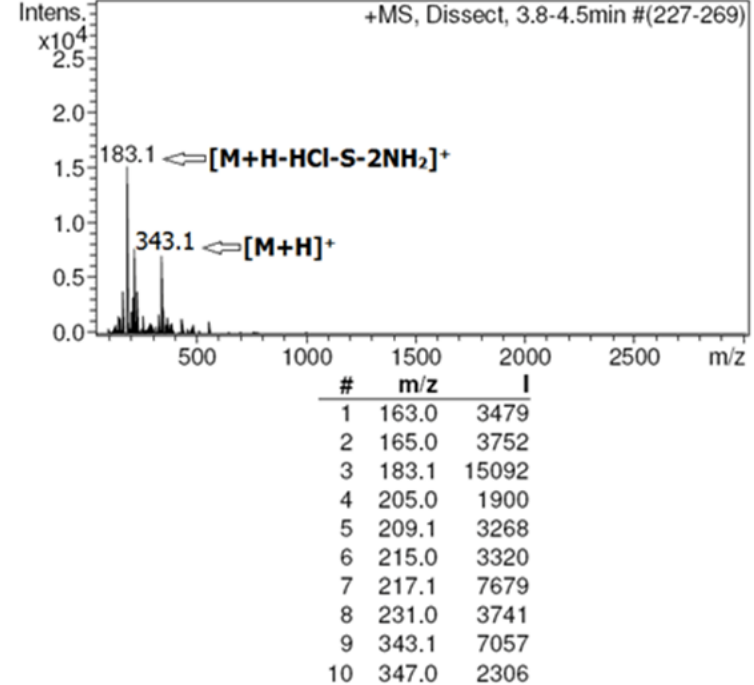

Figure 6 Mass spectra of etizolam at retention time $6.2 \mathrm{~min}$.

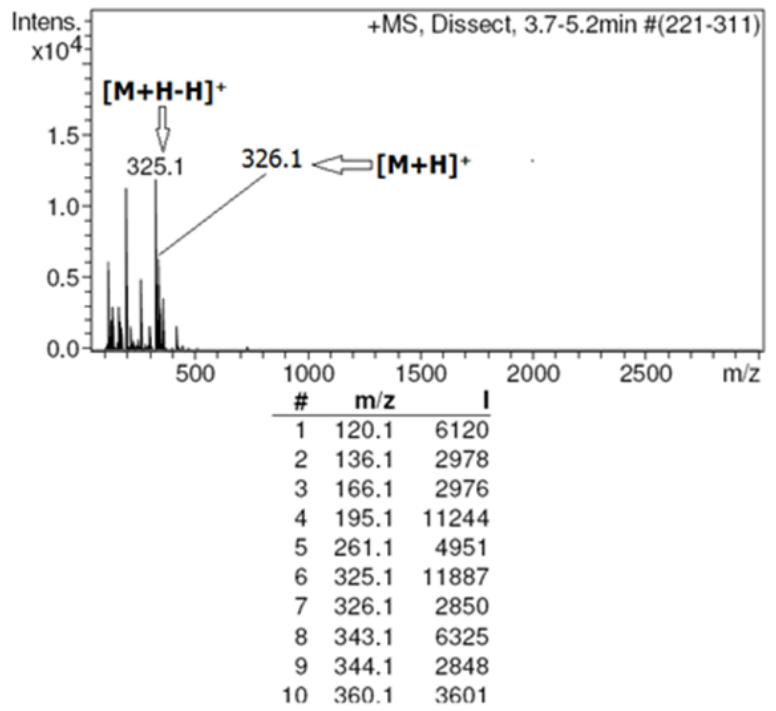

Figure 7 Mass spectra of midazolam at retention time $3.5 \mathrm{~min}$.

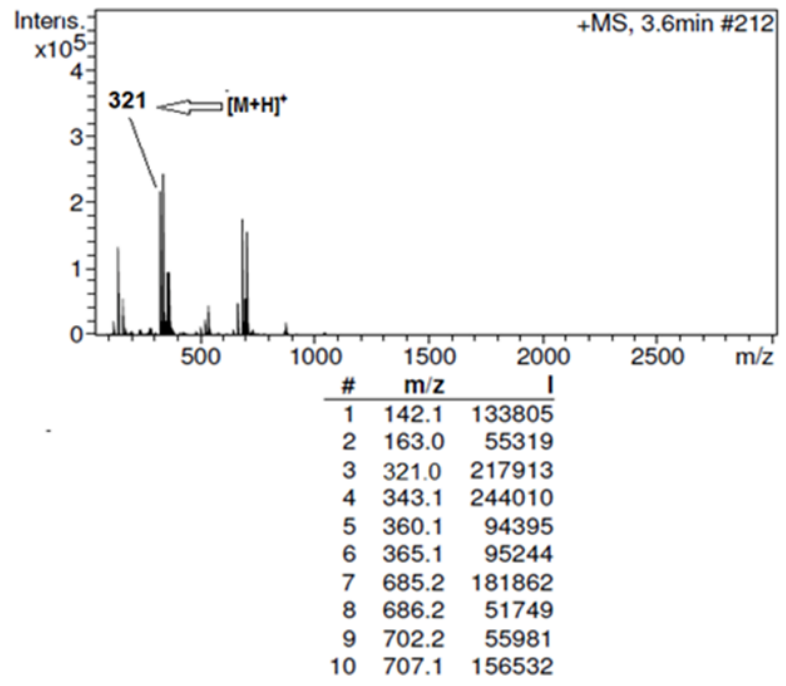

Figure 8 Mass spectra of lorazepam at retention time $3.6 \mathrm{~min}$.

Citation: Thangadurai S, Karthikprabu B, Tamilarasan A. Simultaneous method for the separation and identification of certain benzodiazepine drugs in pharmaceutical formulations by liquid chromatography-tandem mass spectrometry (LC-MS/MS). Forensic Res Criminol Int J. 20I 7;5(I):202-2 I0. DOI: $10.15406 /$ frcij.2017.05.00140 


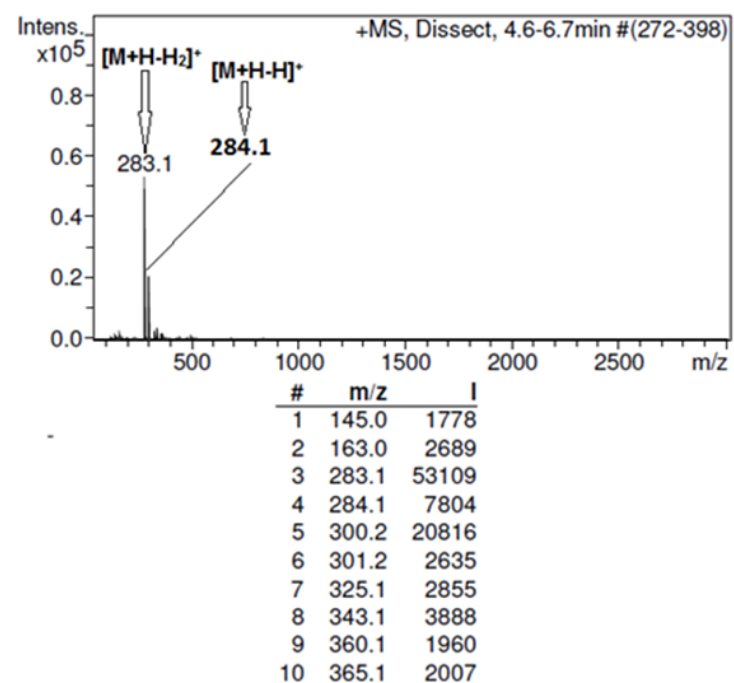

Figure 9 Mass spectra of diazepam at retention time $5.3 \mathrm{~min}$.

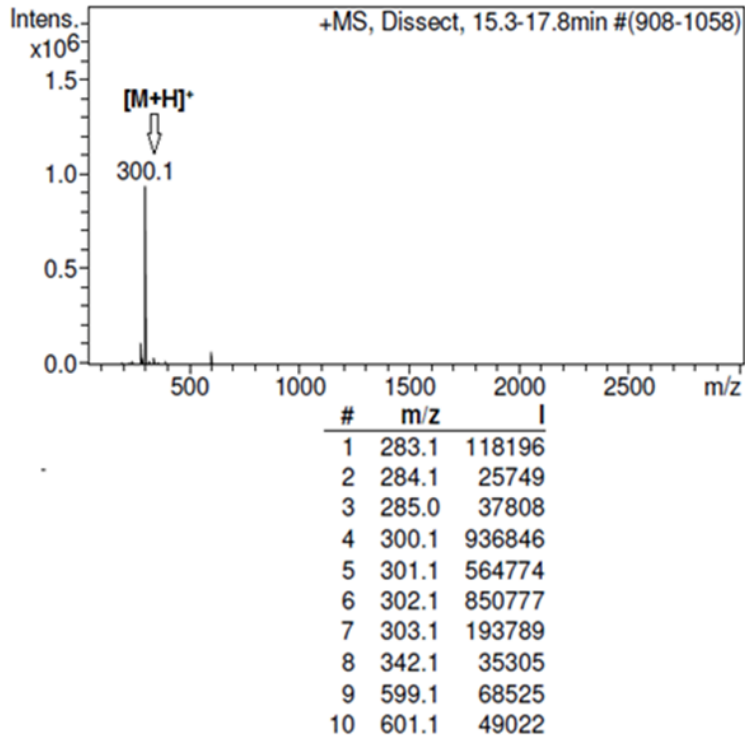

Figure 10 Mass spectra of chlordiazepoxide at retention time $16.0 \mathrm{~min}$.

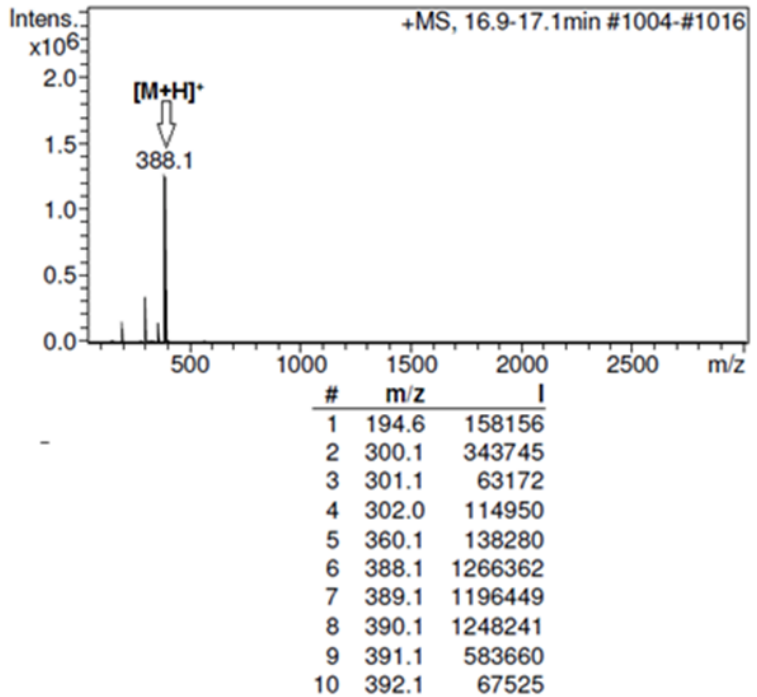

Figure I I Mass spectra of flurazepam at retention time $16.9 \mathrm{~min}$.

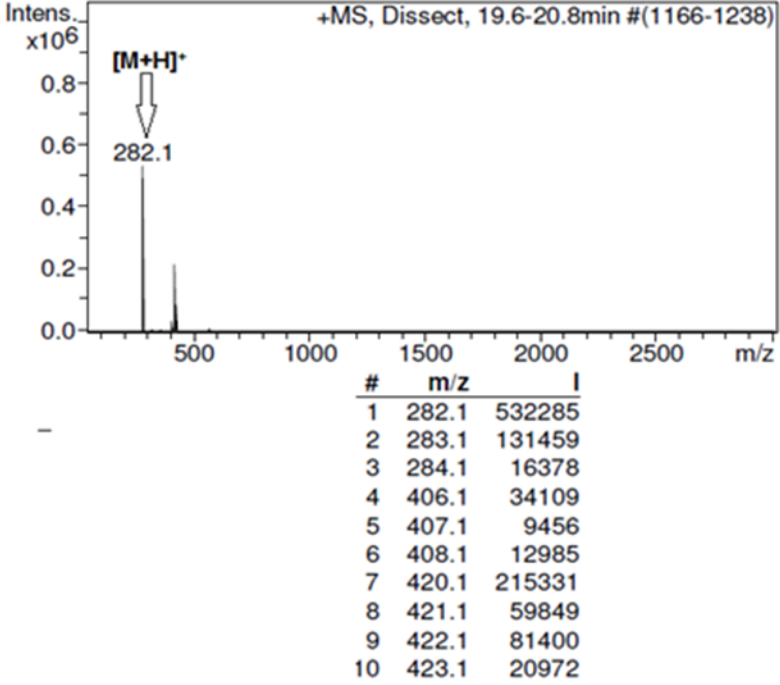

Figure 12 Mass spectra of nitrazepam at retention time $20.1 \mathrm{~min}$.

The optimised conditions for estimation provided a well-defined separation between the drug, internal standard and endogenous components. The blank samples showed no interference at retention time of the drugs and their internal standards. In general, the Limit of Detection (LOD) of compounds detected by MS-MS is lower than the LODs of substances submitted to MS. The LOD value was found to be $250 \mu \mathrm{g} / \mathrm{ml}$ for all BZDs. This observation showed that the developed methods have adequate sensitivity. These values, however, may be affected by the separation conditions (e.g., column, reagents, and instrumentation and data systems), instrumental changes (e.g., pumping systems and detectors) and use of non HPLC grade solvents and may result in changes in signal to noise ratios. The optimized parameters for different BZDs monitored are given in Table 2.

The LC-MS/MS method described in this work allows us to analyze ten BZDs, in pharmaceutical dosage forms and to reach detection limits lower than those observed with conventional LC-DAD method. Low dose as well as high dose BZDs can be determined in one run. The results of this study show the advantages of LC-MS/MS: higher specificity, lower detection limits, simultaneous measurement of many substances as well as satisfactory validation characteristics. The alternation of MS and MS-MS detection allows us to measure high and low levels of different substances simultaneously; no interference has been noticed.

The low limits of detection make this method suitable for the analysis of BZDs in whole samples as well as in other biological fluids such as oral fluid.This may be an advantage in research projects concerning road side drug testing. In other methods described, ${ }^{30}$ the $\mathrm{pH}$ of the mobile phase varied from acidic to slightly alkaline. ${ }^{19}$ In our protocol, best separation was achieved by using a solution at $\mathrm{pH} 3$. A run of $25 \mathrm{~min}$, including a re-equilibration time of $10 \mathrm{~min}$ was selected. Various mobile phase compositions were evaluated, with the objective being a best compromise among a simple LC method, reasonably short run time, and maximizing chromatographic resolution of the analytes. The choices included gradient methods using either $20 \mathrm{mM}$ ammonium formate at $\mathrm{pH} 3$ or $\mathrm{pH} 9$, or $0.1 \%$ formic acid. Once APCI was chosen as the preferred ionization method, methanol become the organic component of choice over acetonitrile, as it provides better sensitivity in APCI and does not build up carbon deposits on the APCI corona needle. Acetonitrile has higher gas phase basicity than methanol; since ionization in APCI occurs in the gas phase (rather

Citation: Thangadurai S, Karthikprabu B, Tamilarasan A. Simultaneous method for the separation and identification of certain benzodiazepine drugs in pharmaceutical formulations by liquid chromatography-tandem mass spectrometry (LC-MS/MS). Forensic Res Criminol Int J. 20I7;5(I):202-2 I0. DOI: $10.15406 /$ frcij.2017.05.00I40 
in the liquid phase as in ESI), acetonitrile can compete with analyte molecules for the available protonation "work".

Optimisation of chromatographic conditions are intended to take into account the various goals of the method development and to weigh each goal (resolutions, run time, sensitivity, peak symmetry, etc) accurately, according to the requirements of HPLC can be used for the estimation of BZDS in pharmaceutical and forensic samples. The optimised conditions for estimation provided a well-defined separation between the drug, internal standard and endogenous components. The blank samples showed no interference at retention time of the drugs and their internal standards. As a result, calibration curves that used relative responses were worse than calibration curves that used absolute responses. No experiments have been performed to optimize the recovery of the internal standard. No significant changes in the chromatographic parameters were observed when changing the experimental conditions (operators, instruments, source of reagents and column of similar type) and optimised conditions $(\mathrm{pH}$, mobile phase ratio and flow rate). System suitability parameters such as column efficiency (theoretical plates), resolution factor and peak asymmetry factor of the optimised methods were found satisfactory.

\section{Conclusion}

In the field of forensic sciences in particular, the use of LC-MS/MS has changed considerably. Where it was once a technique used only infrequently: that is an alternative to GC-MS for more 'troublesome' analyses i.e., highly polar or thermo labile compounds. LC-MS has now emerged as a technique that is a worthy contender for the status of gold standard. The high sensitivity in combination with the ease at which structural analysis can be performed makes LC-MS/ MS a practical problem solving tool. Unlike GC/MS, this technique requires no derivatisation and due to softer ionisation it is possible to monitor specific fragmentation from the protonated molecular species of the analyte. This sensitive and selective method offers the opportunity for simultaneous screening and identification of almost all BZDs which are available in India and that are relevant in clinical and forensic cases. Low dose as well as high dose BZDs can be measured simultaneously, with low detection limits, and satisfactory validation characteristics. Hence, the current investigation concluded by representing the significant analytical work that could be used for the BZDs in separation and detection by LC-MS/MS method in pharmaceutical dosage forms as well as in forensic samples or in the confiscated materials. This method could be potentially applied in the analysis of forensic samples from DFSA cases.

\section{Acknowledgments}

The authors are very grateful to the management, The SASTRA University, Tanjavur, Tamil Nadu, India for their help in the analysis of LC-MS/MS. One of the authors (S.T) greatly acknowledges the UGC-SERO for the financial support to carry out this work.

\section{Conflicts of interest}

None.

\section{References}

1. Brettel TA, Butler JM, Almirall JR. Forensic Science. Anal Chem 2011;83(12):4539-4556.

2. Ngwa G, Fritch D, Blum K, et al. Simultaneous analysis of 14 benzodiazepines in oral fluid by solid-phase extraction and LC-MS-MS. $J$ Anal Toxicol. 2007;31(7):369-376.
3. Al Saffar Y, Stephanson NN, Beck O. Multicomponent LC-MS/MS screening method for detection of new psychoactive drugs, legal highs, in urine-experience from the Swedish population. J Chromatogr B Analyt Technol Biomed Life Sci. 2013;930:112-120.

4. Deveaux M, Chèze M, Pépin G. The role of LC-MS-MS to test blood and urine samples for the toxicological investigation of DFC. Ther Drug Monit. 2008;30(2):225-228.

5. Beynon CM, McVeigh C, McVeigh, et al. The involvement of drugs and alcohol in drug-facilitated sexual assault, a systematic review of the evidence. Trauma Violence Abuse. 2008;9(3):178-188.

6. Cook JM, Marshall R, Masci C, et al. Physician's perspectives on prescribing Benzodiazepines for older adults: A qualitative study. $J$ Gen Intern Med. 20047;22(3):303-307.

7. McCormick SR, Nielsen J, Jatlow PI. Alprazolam overdose: clinical findings and serum concentrations in two cases. $J$ Clin Psychiatry. 1985;46(6):247-328.

8. Kevin C, Honey C, Hart JP. Electrochemical Detection of Benzodiazepines, Following Liquid Chromatography, for Applications in Pharmaceutical, Biomedical and Forensic Investigations. In sciences J. 2014;4(1):1-18.

9. Proencaa P, Teixeiraa H, Pinheiroa J, et al. Forensic intoxication with clobazam: HPLC/DAD/MSD analysis. Forensic Sci Int. 2004;143(23):205-209.

10. Drummer OH, Ranson DL. Sudden death and benzodiazepinas. Am J Forensic Med Patholog. 1996;17:336-342.

11. Bolner A, Tagliaro F, Lomeo A. Optimized determination of clobazam in human plasma with extraction and high-performance liquid chromatography analysis. J Chromatogr B. 2001;750:177-180.

12. Kunicki PK. Simples and sensitive high-performance liquid chromatography method for the determination 1,5-benzodiazepine clobazam and its active metabolite $\mathrm{N}$-desmethylclobazam in human serum and urine with application to 1,4-benzodiazepines analysis. J Chromatogr B Biomed Sci Appl. 2001;750(1):41-49.

13. Gowri Bala Kumari K, S Vanilatha, M Mary Theresa, et al. RP-HPLC Method Development and Validation for the Analysis of Clobazam in Pharmaceutical Dosage forms. Journal of Atoms and Molecules. 2011;1(1):55-63.

14. Gazdzik WR, Podleśny J, Filipek M. HPLC method for simultaneous determination of clobazam and N-desmethylclobazam in human serum, rat serum and rat brain homogenates. Biomed Chromatogr. 1989;3(2):7981.

15. Idris JM, Sudhakar P, Priyankar G, et al. Determination of Clonazepam in chocolate using High Performance Liquid Chromatography and further confirmation by Gas Chromatography-Mass Spectrometry. Asian Journal of Research in Chemistry. 2011;4(5):761-765.

16. de Carvalho D, Lanchote VL. Measurement of plasma clonazepam for therapeutic control: a comparison of chromatographic methods. Ther Drug Monit. 1991;13(1):55-63.

17. Valenza T, Rosselli P. Rapid and specific high-performance liquid chromatographic determination of clonazepam in plasma. J Chromatogr. 1987;386:363-376.

18. Sallustio BC, Kassapidis C, Morris RG. High-performance liquid chromatography determination of clonazepam in plasma using solidphase extraction. Ther Drug Monit. 1994;16(2):174-178.

19. Enein HY, Thiffault C. Rapid liquid chromatographic analysis of flurazepam in pharmaceutical preparation. Toxicological \& Environmental Chemistry. 1992;34(2-4):73-76.

20. Srikantha D, Ramesh Raju RR. New RP-HPLC Method Development and Validation for Flurazepam in Pure and Capsule Dosage Form. Journal of Pharmaceutical Research. 2014;13(1):23-26. 
21. Dadgar D, Smyth WF Hojabri H. High-performance liquid chromatographic determination of flurazepam and its metabolites in human blood plasma. Analytica Chimica Acta. 1983;147:381-385.

22. Selinger K, Lessard D, Hill HM. Simultaneous determination of flurazepam and its metabolites in human plasma by high- performance liquid chromatography. J Chromatogr. 1989;494:247-256.

23. Mehta AC. High-pressure liquid chromatographic determination of some 1, 4-benzodiazepines and their metabolites in biological fluids: A review. Talanta. 1984;31(1):1-8.

24. Ferslew KE, Hagardorn AN, McCormick WF. Postmortem determination of the biological distribution of Sufentanil and Midazolam after an acute intoxication. J Forensic Sci. 1989;34(1):249-257.

25. Dordevic S, Tomasevic G Kilibarda V. Fatal Overdose with MidazolamApplication of HPLC-PDA Method. Med Data Rev. 2010;2(3):251-255.

26. Nishiyama T, Hanaoka K. Accidental overdose of midazolam as intramuscular premedication. J Clin Anesth. 2002;14(7):543-555.

27. Michalodimitrakis M, Christodoulou P, Tsatsakis AM, et al. Death related to midazolam overdose during endoscopic retrograde cholangiopancreatography. Am J Forensic Med Pathol. 1999;20(1):9397.

28. Famiglini G, Termopoli V, Palma P, et al. Liquid chromatography-electron ionization tandem mass spectrometry with the Direct-EI interface in the fast determination of diazepam and flunitrazepam in alcoholic beverages. Electrophoresis. 2016;37(7-8):1048-1054.

29. Xiang P, Sun Q, Shen B, et al. Segmental hair analysis using liquid chromatography-tandem mass spectrometry after a single dose of benzodiazepines. Forensic Sci Int. 2011;204(1-3):19-26.

30. Fisichella M, Morini L, Sempio C, et al. Validation of a multi-analyte LCMS/MS method for screening and quantification of 87 psychoactive drugs and their metabolites in hair. Anal Bioanal Chem. 2014;406(14):34973506 .

31. Miki A, Tatsuno M, Katagi M, et al. Simultaneous determination of eleven benzodiazepine hypnotics and eleven relevant metabolites in urine by column-switching liquid chromatography-mass spectrometry. $J$ Anal Toxicol. 2002;26(2):87-93.
32. MJ Bogusz. Liquid chromatography mass spectrometry as a routine method in forensic sciences: a proof of maturity. J Chromatogr B Biomed Sci Appl. 2000;748(1):3-19.

33. Jeong YD, Kim MK, Suh SI, et al. Rapid determination of benzodiazepines, zolpidem and their metabolites in urine using direct injection liquid chromatography tandem mass spectrometry. Forensic Sci Int. 2015;257:84-92.

34. Lingjuan Xiong, Wang R, Liang C, et al. Determination of co-administrated opioids and benzodiazepines in urine using column-switching solid-phase extraction and liquid chromatography-tandem mass spectrometry. $J$ Chromatogr A. 2015;1395:99-108.

35. Jang M, Chang H, Yang W, et al. Development of an LC-MS/MS method for the simultaneous determination of 25 benzodiazepines and zolpidem in oral fluid and its application to authentic samples from regular drug users. J Pharm and Biomed Anal. 2013;74:213-222.

36. Lee HH, Lee JF, Lin SY, et al. Simultaneous determination of HFBAderivatized amphetamines and ketamines in urine by gas chromatographymass spectrometry. J Anal Toxicol. 2011;35(3):162-169.

37. Strano Rossi S, Molaioni F, Rossi F, et al. Rapid screening of drugs of abuse and their metabolites by gas chromatography/ mass spectrometry: application to urinalysis. Rapid Commun Mass Spectrom. 2005;19(11):1529-1535.

38. Lee HH, Lee JF, Lin SY, et al. Simultaneous quantification of urine flunitrazepam, nimetazepam and nitrazepam by using liquid chromatography tandem mass spectrometry. Clin Chim Acta. 2013;420:134-139.

39. Lips AG, Lameijer W, Fokkens RH, et al. Methodology for the development of a drug library based upon collision-induced fragmentation for the identification of toxicologically relevant drugs in plasma samples. J Chromatogr B Biomed Sci Appl. 2001;759(2):191-207. 